\title{
Goal-oriented treatment and combination therapy for pulmonary arterial hypertension
}

\author{
M.M. Hoeper, I. Markevych, E. Spiekerkoetter, T. Welte and J. Niedermeyer
}

\begin{abstract}
Combination therapy may improve outcome in patients with severe pulmonary arterial hypertension (PAH).

PAH patients were treated according to a goal-oriented therapeutic strategy. Patients who did not reach the treatment goals with monotherapy received combination treatment according to a predefined strategy, including bosentan, sildenafil and inhaled iloprost. Intravenous iloprost and lung transplantation were reserved for treatment failures. End points were overall survival, transplantation-free survival, and survival free from transplantation and intravenous prostanoid treatment.
\end{abstract}

Between January 2002 and December 2004, 123 consecutive patients with PAH were treated according to the novel approach. Survival at 1, 2 and 3 yrs was 93.0, 83.1 and 79.9\%, respectively, which was significantly better than the survival of a historical control group, as well as the expected survival. Compared to the historical control group, the use of combination treatment also significantly improved the combined end point of death, lung transplantation and need for intravenous iloprost treatment.

In conclusion, a therapeutic approach utilising combinations of bosentan, sildenafil and inhaled iloprost in conjunction with a goal-oriented treatment strategy provides acceptable long-term results in patients with severe pulmonary arterial hypertension, and reduces the need for intravenous prostaglandin treatment and lung transplantation.

KEYWORDS: Endothelin receptor antagonists, hypertension, prostaglandins, pulmonary, sildenafil

D) ulmonary arterial hypertension (PAH) is no longer an untreatable disease. Intravenous epoprostenol was the first therapy shown to improve haemodynamics, exercise capacity and survival in patients with this condition [1]. In recent years, several novel prostanoids, including oral beraprost, subcutaneous treprostinil and aerosolised iloprost, have been shown in relatively short-term clinical trials to improve exercise capacity and haemodynamics [2-4]. In addition, the endothelin receptor antagonist bosentan $[5,6]$ and the phosphodiesterase-5-inhibitor sildenafil have enriched the therapeutic arsenal for PAH $[7,8]$.

Current treatment algorithms for $\mathrm{PAH}$ [9-11] recommend bosentan or a nonparenteral prostanoid as first-line treatment for PAH in functional class III, whereas intravenous prostaglandins should be reserved for patients in functional class IV. Sildenafil was not approved for PAH when these guidelines were published, but it is expected that this drug will be used mainly in patients who present in functional class III. Despite the lack of systematic studies, these guidelines also suggest combination therapy as one possible option for patients who respond insufficiently to monotherapy.

The criteria for instituting combination therapy have never been defined. However, retrospective studies may provide some clues as to which variables may be useful for therapeutic decisions. A recent study by SITBON et al. [12] showed that patients who reached a 6-min walk distance of $>380 \mathrm{~m} 3$ months after introduction of epoprostenol treatment, had a significantly better long-term survival than patients who did not reach this threshold [12]. In addition, a study by WENSEL et al. [13] showed that cardiopulmonary exercise testing may also be useful, since patients who had a peak oxygen uptake of $<10.4 \mathrm{~mL} \cdot \mathrm{min}^{-1} \cdot \mathrm{kg}^{-1}$ and a maximum systolic blood pressure during exercise $<120 \mathrm{mmHg}$ had a much worse prognosis than patients who were able to meet these thresholds [13]. This study selected the same criteria to guide therapeutic decisions, because of their noninvasive accessibility and their prognostic importance.
AFFILIATIONS

Dept of Respiratory Medicine, Hannover Medical School, Hannover, Germany.

CORRESPONDENCE

M.M. Hoeper

Hannover Medical School

Dept of Respiratory Medicine

Carl-Neuberg-Str. 1

30625 Hannover

Germany

Fax: 495115328536

E-mail: hoeper.marius@mh-

hannover.de

Received:

June 272005

Accepted after revision:

July 262005 
This paper describes the 3-yr experience with this approach, comparing survival of patients treated between January 2002 and December 2004 with the expected survival and with the historical survival of PAH patients treated between January 1999 and December 2001, i.e. before bosentan and sildenafil became available.

\section{METHODS}

Hannover Medical School (Hannover, Germany) is a referral centre for patients with pulmonary hypertension. Patients are regularly seen in the outpatient clinic at 1-6 monthly intervals depending on clinical stability. Follow-up examinations include clinical and functional assessment, regular 6-min walk tests and cardiopulmonary exercise testing.

Patients with $\mathrm{PAH}$ were/are treated according to predefined algorithms, as follows.

1) Before 2002, the therapeutic strategy for patients with $\mathrm{PAH}$ in functional class III or IV without haemodynamic instability was to institute either beraprost or inhaled iloprost as first-line treatment. Patients in functional class IV with haemodynamic instability were immediately treated with intravenous iloprost. Patients with progressive disease despite the use of nonparenteral prostanoids (i.e. those who progressed to functional class IV and/or developed signs of right heart failure unresponsive to diuretics) were switched to intravenous iloprost and listed for lung transplantation, if eligible.

2) When bosentan and sildenafil became available in January 2002, the treatment strategy for PAH was revisited. For the reasons discussed previously, the following treatment goals were defined: a 6-min walking distance $>380 \mathrm{~m}$, a peak oxygen uptake $>10.4 \mathrm{~mL} \cdot \mathrm{min}^{-1} \cdot \mathrm{kg}^{-1}$ and a peak systolic blood pressure during exercise $>120 \mathrm{mmHg}$. Patients were considered clinically stable when all three treatment goals were reached. For patients with newly diagnosed PAH in functional class III and IV, bosentan was considered first-line treatment. In patients who did not reach one or more of these treatment goals on two consecutive visits, combination therapy was initiated according to a predefined algorithm (fig. 1). When treatment goals were not met with bosentan alone, sildenafil was the preferred combination partner, followed by the addition of inhaled iloprost, if needed. Intravenous iloprost and lung transplantation were considered only for patients not reaching the predefined goals with triple combination treatment.

To be eligible for this analysis, patients had to suffer from PAH in functional class III or IV before institution of medical therapy. Patients with other variants of pulmonary hypertension, including pulmonary veno-occlusive disease, were excluded, as were patients in functional class I or II at baseline, and patients with a positive response to acute vasodilator challenge who received treatment with high-dose calcium channel blockers.

Patients were always informed about all available treatment options. Whenever combination treatment was proposed, patients were informed that this kind of therapy, regardless of the combinations used, was not scientifically evaluated and that potential risks or side-effects could not be excluded. In addition, patients were always informed about the possibility

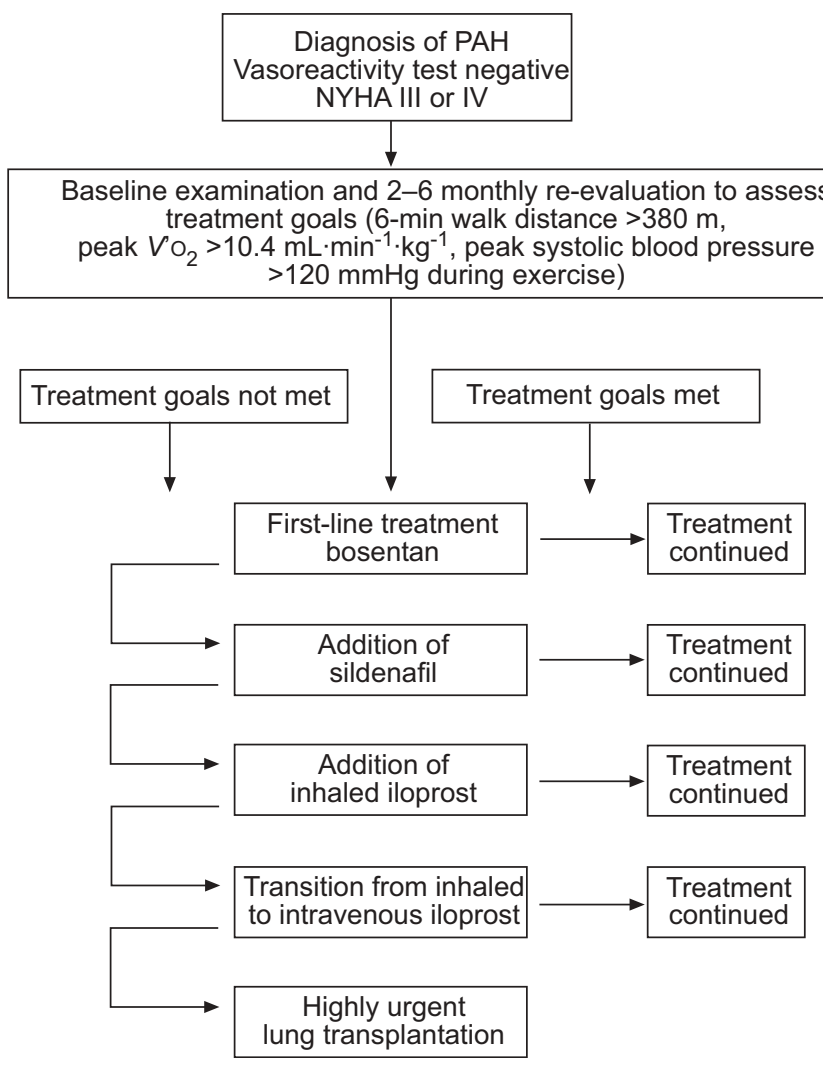

FIGURE 1. Therapeutic strategy for patients with newly diagnosed pulmonary arterial hypertension $(\mathrm{PAH})$ not requiring immediate treatment with intravenous iloprost. NYHA: New York Heart Association; $V^{\prime} \mathrm{O}_{2}$ : oxygen uptake.

of intravenous iloprost treatment. The local ethics committee approved this protocol.

\section{Dosing regimens}

Bosentan was given at a dose of $62.5 \mathrm{mg}$ b.i.d. for 4 weeks, followed by $125 \mathrm{mg}$ b.i.d. thereafter [5]. Liver function tests were performed at 4-week intervals, and elevations of transaminases were handled according to the bosentan summary of product characteristics. Sildenafil was started at $25 \mathrm{mg} \mathrm{3-4}$ times daily and was titrated up to $50 \mathrm{mg}$ t.i.d. in patients with an insufficient response to the initial dose [14]. Inhaled iloprost was administered at a dose of $6 \times 5 \mu \mathrm{g} \cdot \mathrm{day}^{-1}$ using an ultrasonic nebuliser (Optineb ${ }^{\circledR}$; Nebutec, Elsenfeld, Germany) [3]. Intravenous iloprost was started at $0.5 \mathrm{ng} \cdot \mathrm{kg}^{-1} \cdot \mathrm{min}^{-1}$ and was gradually titrated based on clinical efficacy and side-effects. The same dosing regimens were used for combination therapy, and drug doses were kept unchanged when a new substance was added.

The same dosing regimens for inhaled and intravenous iloprost were also used in the historical control group. Beraprost was started at $20 \mu \mathrm{g}$ q.i.d. and was increased by 20 $\mu \mathrm{g}$ q.i.d. each week to a target dose of $120 \mu \mathrm{g}$ q.i.d. or until the maximum tolerated dose was reached.

\section{Statistical analysis}

All data are expressed as mean $\pm \mathrm{SD}$. Differences between the two treatment groups (1999-2001 and 2002-2004) were assessed by Chi-squared analysis for categorical variables 
and by Mann-Whitney rank-sum test for continuous variables. Survival, transplantation-free survival and the combined end point "survival free from transplantation and intravenous prostaglandin treatment" were estimated using the KaplanMeier method, and any differences were evaluated with a stratified log-rank test. Chi-squared analysis was used to compare the observed survival rates at 1, 2 and 3 yrs with the expected survival rates. Expected survival (idiopathic (I)PAH only) was calculated according to the National Institutes of Health (NIH) equation $[15,16]$, with the following:

$$
\mathrm{P}(\mathrm{t})=\mathrm{H}(\mathrm{t})^{\mathrm{A}(\mathrm{x}, \mathrm{y}, \mathrm{z})}
$$

with

$$
\mathrm{A}(\mathrm{x}, \mathrm{y}, \mathrm{z})=\mathrm{EXP}(0.007325 \mathrm{x}+0.0526 \mathrm{y}-0.3235 \mathrm{z})
$$

where $\mathrm{x}$ is mean pulmonary arterial pressure, $\mathrm{y}$ is mean right atrial pressure and $\mathrm{z}$ is cardiac index. The probabilities of survival at 1,2 and $3 \mathrm{yrs}$ are $\mathrm{P} 1=0.75^{\mathrm{A}}, \mathrm{P}_{2}=0.65^{\mathrm{A}}$ and $\mathrm{P}_{3}=0.55^{\mathrm{A}}$. A p-value $<0.05$ was considered statistically significant.

\section{RESULTS}

Between January 2002 and December 2004, a total of 135 patients with PAH were referred to the Hannover Medical School. Twelve patients were excluded, either because they were in functional class NYHA I or II without specific treatment $(n=4)$, or because they fulfilled the criteria as calcium channel blocker responders $(n=8)$. Thus, 123 patients with PAH in NYHA functional class III or IV were eligible for this analysis. The baseline characteristics of these patients are shown in table 1.

\section{Medication and outcome}

Overall, 17 out of $123 \mathrm{PAH}$ patients (13.8\%) died during the observation period. The probability of survival was 93.0, 83.1 and $79.9 \%$ at 1,2 and 3 yrs, respectively (fig. 2a). Causes of death were right heart failure $(n=10)$, sudden death $(n=3)$, respiratory failure due to pneumonia $(n=1)$ and other intercurrent illnesses $(n=3)$.

A total of 118 patients were started on monotherapy. In order to reach the predefined treatment goals, the introduction of a two-drug regimen became necessary in 51 patients $(43.2 \%)$. Nineteen patients $(16.1 \%)$ eventually required a triple-drug regimen, including bosentan, sildenafil and inhaled iloprost. Five patients $(4.2 \%)$ had to be switched to intravenous iloprost treatment, and one patient required lung transplantation.

Five patients were started on intravenous iloprost because they initially presented in functional class IV with haemodynamic instability. Of these patients, two died; the other three improved, and two of them were changed from intravenous iloprost to a combination of bosentan and sildenafil and remained stable throughout the observation period (13 months and 24 months, respectively). In one of these patients, switching became necessary after three consecutive episodes of line infection with life-threatening septicaemia. This patient was otherwise stable in functional class III with 6-min walk distances between $350 \mathrm{~m}$ and $400 \mathrm{~m}$ both before and after transfer from intravenous iloprost to bosentan/sildenafil

\begin{tabular}{|c|c|c|c|}
\hline \multirow[t]{2}{*}{ TABLE 1} & racteristics of $t$ & the patient & \multirow[b]{2}{*}{ p-value } \\
\hline & $\begin{array}{l}\text { Population } \\
\text { under study }\end{array}$ & $\begin{array}{l}\text { Historical } \\
\text { control group }\end{array}$ & \\
\hline Subjects $n$ & 123 & 84 & \\
\hline Female/male & 90/33 (73.2/26.8) & $57 / 27(67.9 / 32.1)$ & 0.41 \\
\hline Age yrs & $52 \pm 14$ & $44 \pm 13$ & $<0.001$ \\
\hline \multicolumn{4}{|l|}{ Diagnosis } \\
\hline IPAH & $89(72)$ & $67(80)$ & $0.23^{\#}$ \\
\hline PAH-CTD & $15(12.2)$ & $5(6.0)$ & \\
\hline $\mathrm{PAH}-\mathrm{CHD}$ & $5(6.5)$ & $3(3.6)$ & \\
\hline PAH-HIV & $2(1.6)$ & $2(3.6)$ & \\
\hline PPHT & $12(9.8)$ & $7(8.3)$ & \\
\hline Functional NYHA class III/IV & 98/25 (79.7/20.3) & $66 / 18(78.6 / 21.4)$ & 0.85 \\
\hline History of syncope & $26(21.1)$ & $16(19.0)$ & 0.71 \\
\hline History of right heart failure & $35(28.5)$ & $22(26.2)$ & 0.72 \\
\hline 6-min walk distance $\mathrm{m}$ & $308 \pm 133$ & $314 \pm 125$ & 0.97 \\
\hline Peak $V^{\prime} \mathrm{O}_{2} \mathrm{~mL} \cdot \mathrm{min}^{-1} \cdot \mathbf{k g}^{-1}$ & $12.1 \pm 3.5$ & NA & NA \\
\hline \multicolumn{4}{|l|}{ Baseline haemodynamics } \\
\hline RAP $\mathrm{mmHg}$ & $8 \pm 5$ & $8 \pm 5$ & 0.81 \\
\hline PAPm mmHg & $52 \pm 10$ & $55 \pm 13$ & 0.15 \\
\hline PCWP mmHg & $7 \pm 3$ & $8 \pm 2$ & 0.30 \\
\hline $\mathrm{CO} \mathrm{L} \cdot \mathrm{min}^{-1}$ & $3.9 \pm 1.2$ & $3.7 \pm 1.2$ & 0.34 \\
\hline $\mathrm{Cl} \mathrm{L} \cdot \mathrm{min}^{-1} \cdot \mathrm{m}^{-2}$ & $2.1 \pm 0.6$ & $2.1 \pm 0.6$ & 0.44 \\
\hline PVR dyn $\cdot s \cdot \mathrm{cm}^{-5}$ & $1027 \pm 443$ & $1122 \pm 465$ & 0.10 \\
\hline $\mathrm{Sv}_{1} \mathrm{O}_{2} \%$ & $61 \pm 9$ & $61 \pm 10$ & 0.88 \\
\hline \multicolumn{4}{|l|}{ Concomitant medication } \\
\hline Oral anticoagulants & $107(88.0)$ & $75(89.3)$ & 0.62 \\
\hline Diuretics & $102(82.9)$ & $72(85.7)$ & 0.59 \\
\hline Digitalis & $23(18.7)$ & $18(21.4)$ & 0.63 \\
\hline \multicolumn{4}{|l|}{ Probability of survival } \\
\hline $1 \mathrm{yr}$ & $0.71 \pm 0.10$ & $0.70 \pm 0.10$ & 0.93 \\
\hline $2 \mathrm{yrs}$ & $0.59 \pm 0.12$ & $0.58 \pm 0.12$ & 0.87 \\
\hline $3 \mathrm{yrs}$ & $0.50 \pm 0.14$ & $0.49 \pm 0.14$ & 0.87 \\
\hline
\end{tabular}
(follow-up 24 months after switching). The other patient had
Data are presented as $\mathrm{n}(\%)$ or mean $\pm \mathrm{SD}$. The time period for the population under study was between January 12002 and December 31 2004. The time period for the historical control group was between January 11999 and December 31 2001. IPAH: idiopathic pulmonary arterial hypertension; PAHCTD: pulmonary arterial hypertension associated with connective tissue disease; PAH-CHD: pulmonary arterial hypertension associated with congenital heart disease; PAH-HIV: pulmonary arterial hypertension associated with human immunodeficiency virus infection; PPHT: portopulmonary hypertension; NYHA: New York Heart Association; peak $V^{\prime} \mathrm{O}_{2}$ : peak oxygen uptake determined by cardiopulmonary exercise testing; RAP: right atrial pressure; PAPm: mean pulmonary arterial pressure; PCWP: pulmonary capillary wedge pressure; $\mathrm{CO}$ : cardiac output; $\mathrm{Cl}$ : cardiac index; PVR: pulmonary vascular resistance; $\mathrm{Sv}_{\mathrm{O}} \mathrm{O}_{2}$ : mixed-venous oxygen saturation; NA: not assessed. ${ }^{\#}$ : IPAH versus non-IPAH only; ": PAH only.

dramatic clinical improvement with intravenous iloprost, but requested to be changed to oral treatment. This patient's functional status improved from class IV at the initial presentation to class I-II with 6-min walk distances $>500 \mathrm{~m}$ after 9 months of intravenous iloprost treatment. The clinical situation remained excellent and unchanged after transfer to bosentan/sildenafil treatment (follow-up 13 months). 

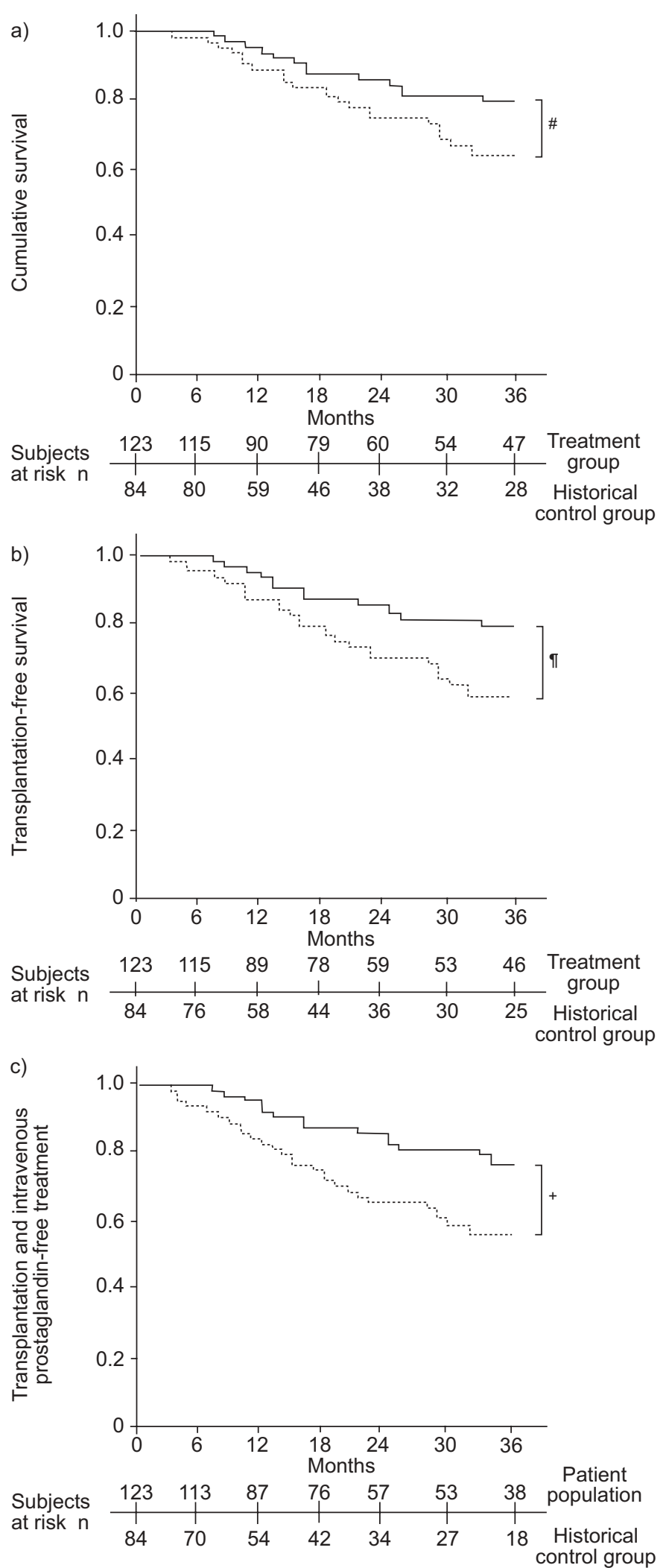

FIGURE 2. Kaplan-Meier estimates of survival of 123 patients with pulmonary arterial hypertension with goal-oriented treatment $(-)$ compared to a historical control group (.......). a) Comparison of cumulative survival. b) Comparison of transplantation-free survival. c) Comparison of survival without transplantation or intravenous prostaglandin treatment. ${ }^{\#}: p=0.047 ;{ }^{\natural}: p=0.007 ;^{+}: p=0.002$.

\section{Comparison with historical control group}

To address the impact of the introduction of novel drugs (i.e. bosentan and sildenafil) and the new treatment strategy on outcome measurements, the PAH patients treated between January 2002 and December 2004 were compared with all class III/IV PAH patients treated between January 1999 and December 2001. As shown in table 1, except for age, both patient groups were well matched. Out of the $84 \mathrm{PAH}$ patients treated in the 1999-2001 period, 20 patients $(23.8 \%)$ died, twelve patients $(14.3 \%)$ required treatment with intravenous iloprost and five patients $(6.0 \%)$ received a lung transplant. The probability of survival at 1, 2 and 3 yrs was $89.8,75.2$ and $63.1 \%$, respectively.

The survival of patients treated between January 2002 and December 2004 was significantly better than that of patients treated in the 1999-2001 period ( $p=0.047$ by Kaplan-Meier analysis; fig. 2a). In addition, transplantation-free survival was superior in the $2002-2004$ cohort ( $\mathrm{p}=0.007$ by Kaplan-Meier analysis; fig. 2b), and there was also a highly significant difference in the combined end point of survival without transplantation or intravenous prostaglandin treatment $(p=0.002$ by Kaplan-Meier analysis; fig. $2 c)$.

\section{Comparing observed and predicted survival (IPAH only)}

The NIH registry equation for predicting survival has been developed and validated only for patients with IPAH $[15,16]$. Therefore, a subgroup analysis of this patient population was performed, comparing observed and expected survival.

The probability of survival of patients with IPAH from 2002$2004(\mathrm{n}=89)$ at 1,2 and 3 yrs was $93.3,88.3$ and $83.9 \%$, respectively, which was significantly better than the survival of IPAH patients in the historical control group (90.9, 74.9 and $60.2 \%$, respectively; $\mathrm{p}=0.011$ by log-rank analysis), and also significantly better than the predicted survival based on the $\mathrm{NIH}$ equation, which was $71.5 \%$ at $1 \mathrm{yr}, 59.9 \%$ at $2 \mathrm{yrs,}$ and $50.0 \%$ at $3 \mathrm{yrs}$, respectively ( $\mathrm{p}<0.001$ for all time points, Chi-squared analysis; fig. 3).

\section{Side-effects}

Bosentan had to be permanently discontinued in five patients secondary to elevations in liver transaminases. Four of these patients were switched to sildenafil therapy and one to inhaled iloprost. Combination therapy was well tolerated by all patients. None of the patients showed signs or symptoms suggestive of additive toxicity of the medications used, as sideeffects were usually attributed to a single drug. Elevations in liver enzymes were attributed to bosentan or heartburn to sildenafil. Symptoms suggesting hypotension more severe than mild orthostatic dysregulation were not observed. Syncope occurred only in patients who had experienced syncope prior to the institution of treatment and was judged as an indication of insufficient treatment rather than as a side-effect of combination therapy. None of the deaths was attributed to medical treatment.

\section{DISCUSSION}

This study addressed the effect of a primarily noninvasive therapeutic concept on survival and other relevant outcome measurements in patients with PAH. Bosentan was used as first-line treatment, and then sildenafil and inhaled iloprost 


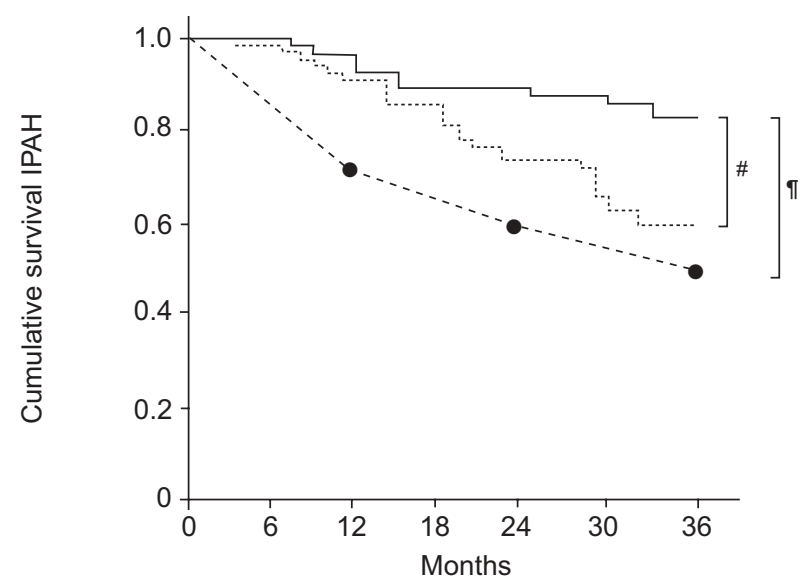

FIGURE 3. Kaplan-Meier estimates of survival of 89 patients with idiopathic pulmonary arterial hypertension (IPAH) with goal-oriented treatment $(-)$ compared with a historical control group $(\cdots \cdots \cdots)$ and with the expected survival (------). ${ }^{\#}$ : $\mathrm{p}=0.011 ;{ }^{* *}: \mathrm{p}<0.001$ for all time points.

were added in a predefined order when the current therapy was not sufficiently effective. To stratify therapeutic decisions, treatment goals were set according to established prognostic criteria: a 6-min walking distance $>380 \mathrm{~m}$; a peak oxygen uptake $>10.4 \mathrm{~mL} \cdot \mathrm{min}^{-1} \cdot \mathrm{kg}^{-1}$; and a peak systolic blood pressure during exercise $>120 \mathrm{mmHg}[12,13]$. Intravenous iloprost treatment or lung transplantation was considered only for patients not reaching these goals with combination therapy. With this approach, the survival rates at 1,2 and 3 yrs were $\sim 93,83$ and $80 \%$, respectively. These results compared favourably with a historical control group of $\mathrm{PAH}$ patients treated before 2002 (i.e. before bosentan and sildenafil became available) and also with the expected survival as calculated from the NIH equation (for IPAH patients only).

Until recently, intravenous epoprostenol was the only treatment that had been shown to improve long-term survival in patients with IPAH. In two large series of patients treated with epoprostenol, 1-yr survival was 85 and $88 \%$, respectively, 2-yr survival was 70 and $76 \%$, respectively, and 3 -yr survival was 63 and 63\%, respectively [12, 17]. The long-term effect of epoprostenol in other forms of $\mathrm{PAH}$ is less well known. On initial observations, the data presented here compare favourably with the results achieved with epoprostenol treatment; however, the patient population differed substantially from those reported by MCLAUGHLIN et al. [17] as well as SITBON et al. [12]. These two series studied only patients with IPAH, whereas the present series included patients with all variants of PAH (although $72 \%$ of these patients suffered from IPAH). Furthermore, the patients presented by MCLAUGHLIN et al. [17], as well as SITBON et al. [12], had more advanced disease at baseline, making any direct comparison of these studies impossible.

Favourable survival rates with first-line bosentan therapy have been reported in another paper by MCLAUGHLIN et al. [6]. In this study of 169 patients with IPAH, survival rates at 1,2 and 3 yrs were 96,89 and $86 \%$, respectively. Three patients (2\%) underwent lung transplantation and 39 patients $(23 \%)$ required addition of, or transfer to, prostacyclin treatment. Again, a direct comparison of these results with the present study is hampered by the different patient populations (all types of PAH versus IPAH only) and the fact that the patients in the present study had more severe disease at baseline. Nevertheless, both studies support the concept of starting $\mathrm{PAH}$ treatment with a single (oral) agent followed by the addition of, or transfer to, a different agent in case of an insufficient clinical result as suggested in the current treatment algorithms [9-11].

It is an important finding of this study that, in order to reach the predefined treatment goals, combination treatment eventually became necessary in almost half of the patients, indicating that monotherapy may not exert sufficient efficacy in numerous patients with PAH. This finding was not unexpected, since none of the currently available treatments offer a cure from the disease, and PAH eventually progresses in many patients despite active therapy. Thus, there is clearly room for further improvement, but the results suggest that a primarily noninvasive treatment strategy and the use of combination treatment may yield acceptable results in the majority of patients with PAH.

The present study has several limitations as follows. A control group treated with intravenous prostaglandins in parallel was not included, making any direct comparison impossible. Furthermore, the treatment goals may not have been optimal. For instance, the 6-min walk distance depends not only on cardiopulmonary fitness but also on other factors, including sex, body weight and height [18]. Thus, a 6-min walk threshold based on predicted values might have been more appropriate than setting an absolute value of $380 \mathrm{~m}$. In addition, serum markers, such as brain natriuretic peptide, may have been added to the list of treatment goals $[19,20]$, but their reliability for therapeutic decision making still needs to be determined. One may also advocate regular invasive monitoring, i.e. right heart catheter examinations, but the current authors prefer to reserve invasive follow-up measurements for unclear situations or difficult therapeutic decisions.

It must also be emphasised that this study did not address the superiority of one treatment over another. The strategy was based on many factors, including published data, personal experience, practicability and economical considerations. Choices of treatment, as well as their order, dosing and timing, have been somewhat arbitrary, and a different approach, for example, using sildenafil or a prostanoid as first-line treatment, may have yielded similar or even better results. In addition, pharmacokinetic interactions between bosentan and sildenafil may be a matter of concern. Sildenafil was recently approved in the USA at a dose of $20 \mathrm{mg}$ t.i.d. However, coadministration of bosentan decreases the plasma concentration of sildenafil by $50-60 \%$ [21], which may have been partly responsible for the fact that almost half of the patients in the present study required sildenafil dosages of $50 \mathrm{mg}$ t.i.d. Further studies are urgently required to address the clinical consequences of these interactions.

Finally, this study does not answer the question of whether combination therapy is truly superior to monotherapy. In patients not reaching the treatment goals with monotherapy, it is obvious that therapy needs to be modified. However, it is 
possible that switching to an alternative treatment could be as efficacious, and considerably less costly, than combination therapy. Prospective, controlled clinical trials are necessary to address this question.

In conclusion, a goal-oriented therapeutic approach utilising currently available treatment options provided reasonably favourable survival rates in patients with severe pulmonary arterial hypertension, while reserving intravenous prostaglandin treatment and lung transplantation for a small population of patients with refractory illness. Further studies are needed to define the variables most useful for clinical decision making and the treatment concepts that provide the best long-term results.

\section{REFERENCES}

1 Barst RJ, Rubin LJ, Long WA, et al. A comparison of continuous intravenous epoprostenol (prostacyclin) with conventional therapy for primary pulmonary hypertension. The Primary Pulmonary Hypertension Study Group. N Engl J Med 1996; 334: 296-302.

2 Galie N, Humbert M, Vachiery JL, et al. Effects of beraprost sodium, an oral prostacyclin analogue, in patients with pulmonary arterial hypertension: a randomized, doubleblind, placebo-controlled trial. J Am Coll Cardiol 2002; 39: 1496-1502.

3 Olschewski H, Simonneau G, Galie N, et al. Inhaled iloprost for severe pulmonary hypertension. $N$ Engl J Med 2002; 347: 322-329.

4 Simonneau G, Barst RJ, Galie N, et al. Continuous subcutaneous infusion of treprostinil, a prostacyclin analogue, in patients with pulmonary arterial hypertension: a double-blind, randomized, placebo-controlled trial. Am J Respir Crit Care Med 2002; 165: 800-804.

5 Rubin LJ, Badesch DB, Barst RJ, et al. Bosentan therapy for pulmonary arterial hypertension. $N$ Engl J Med 2002; 346: 896-903.

6 McLaughlin VV, Sitbon O, Badesch DB, et al. Survival with first-line bosentan in patients with primary pulmonary hypertension. Eur Respir J 2005; 25: 244-249.

7 Michelakis E, Tymchak W, Lien D, Webster L, Hashimoto K, Archer S. Oral sildenafil is an effective and specific pulmonary vasodilator in patients with pulmonary arterial hypertension: comparison with inhaled nitric oxide. Circulation 2002; 105: 2398-2403.

8 Sastry BK, Narasimhan C, Reddy NK, Raju BS. Clinical efficacy of sildenafil in primary pulmonary hypertension: a randomized, placebo-controlled, double-blind, crossover study. J Am Coll Cardiol 2004; 43: 1149-1153.
9 Galie N, Seeger W, Naeije R, Simonneau G, Rubin LJ. Comparative analysis of clinical trials and evidence-based treatment algorithm in pulmonary arterial hypertension. $J$ Am Coll Cardiol 2004; 43: Suppl. 12, 81S-88S.

10 Badesch DB, Abman SH, Ahearn GS, et al. Medical therapy for pulmonary arterial hypertension: ACCP evidence-based clinical practice guidelines. Chest 2004; 126: Suppl. 1, 35S-62S.

11 Galie N, Torbicki A, Barst R, et al. Guidelines on diagnosis and treatment of pulmonary arterial hypertension. The Task Force on Diagnosis and Treatment of Pulmonary Arterial Hypertension of the European Society of Cardiology. Eur Heart J 2004; 25: 2243-2278.

12 Sitbon O, Humbert M, Nunes $\mathrm{H}$, et al. Long-term intravenous epoprostenol infusion in primary pulmonary hypertension: prognostic factors and survival. J Am Coll Cardiol 2002; 40: 780-788.

13 Wensel R, Opitz CF, Anker SD, et al. Assessment of survival in patients with primary pulmonary hypertension: importance of cardiopulmonary exercise testing. Circulation 2002; 106: 319-324.

14 Hoeper MM, Faulenbach C, Golpon H, Winkler J, Welte T, Niedermeyer J. Combination therapy with bosentan and sildenafil in idiopathic pulmonary arterial hypertension. Eur Respir J 2004; 24: 1007-1010.

15 D'Alonzo GE, Barst RJ, Ayres SM, et al. Survival in patients with primary pulmonary hypertension. Results from a national prospective registry. Ann Intern Med 1991; 115: 343-349.

16 Sandoval J, Bauerle O, Palomar A, et al. Survival in primary pulmonary hypertension. Validation of a prognostic equation. Circulation 1994; 89: 1733-1744.

17 McLaughlin VV, Shillington A, Rich S. Survival in primary pulmonary hypertension: the impact of epoprostenol therapy. Circulation 2002; 106: 1477-1482.

18 Enright PL, Sherrill DL. Reference equations for the sixminute walk in healthy adults. Am J Respir Crit Care Med 1998; 158: 1384-1387.

19 Nagaya N, Nishikimi T, Okano Y, et al. Plasma brain natriuretic peptide levels increase in proportion to the extent of right ventricular dysfunction in pulmonary hypertension. J Am Coll Cardiol 1998; 31: 202-208.

20 Leuchte $\mathrm{HH}$, Holzapfel M, Baumgartner RA, et al. Clinical significance of brain natriuretic peptide in primary pulmonary hypertension. J Am Coll Cardiol 2004; 43: 764-770.

21 Paul GA, Gibbs JS, Boobis AR, Abbas A, Wilkins MR. Bosentan decreases the plasma concentration of sildenafil when coprescribed in pulmonary hypertension. $\mathrm{Br} \mathrm{J} \mathrm{Clin}$ Pharmacol 2005; 60: 107-112. 